\title{
A Recurrent Abdominal Pain with Chronic Constipation
}

\author{
Malcotti Federica ${ }^{1}$, Scintu Ambra ${ }^{1}$, Nieri Stefano ${ }^{2}$ and Parmigiani Stefano ${ }^{3 *}$ \\ ${ }^{1}$ General Practitioner Trainee, Eastern Liguria Hospital Sant'Andrea, Italy \\ ${ }^{2}$ Pediatrician, Senior Consultant, Eastern Liguria Hospital Sant'Andrea, Italy \\ ${ }^{3}$ Chief, Mother. Infant Department, Eastern Liguria Hospital Sant'Andrea, Italy
}

\begin{abstract}
We here report the case of a 5-years-old child with recurrent abdominal pain and chronic constipation. For that reason, the baby came to our hospital several times with symptoms' resolution after enema. Despite the medical therapy and the instrumental approaches, the situation of the last admission proceeded to intestinal obstruction and therefore to specialistic surgery in emergency during the night, with resolution of the obstruction that was due to a congenital malformation that was gone undiagnosed during pregnancy, at birth and during the previous admissions. Such anomaly is rarely diagnosed after the neonatal period, anyway the present case underlines that this condition must be taken into account in every patient with recurrent abdominal pain and constipation episodes.
\end{abstract}

Keywords

Congenital malformation, Recurrent abdominal pain, Constipation

\section{Introduction}

Male child, 5-years-old, was referred to our pediatric ER for vomiting and left iliac fossa abdominal pain. The baby had had at least two previous admissions for colicky pain. He suffered from constipation and the other admissions had ended with evacuation after enema. Plain abdomen $X$-rays had seen distention of transverse and right colon, and normal left colon. In the present admission the pain was stronger than before, but always colicky in type. In the history he was born at term by caesarean section and constipation was present since the age of 3 . In the family history the mother was affected by celiac disease.

\section{Case Report}

At the admission the patient was suffering but afebrile. At physical examination the abdomen was treatable on all quadrants, painful to superficial and deep palpation in the left iliac fossa with the presence of colic cord. There were no signs of peritonism. Peristalsis was present and valid. Immediately abdominal ultrasounds were performed: There were no free liquid collections in the abdominal cavity and intestinal loops were not thickened, no signs of volvulus nor intussusception were present. Hypochondriac organs and urinary tract were normal. The urine multi stick test was positive for ketones. Blood tests in emergency showed: WBC 11,600, N 82.7\%, L 12.3\%. Hb 12 g/dL, CRP $0.196 \mathrm{mg} / \mathrm{dL}$.

I.v. rehydratation with normal saline, acetaminophene and $n$-butylbromide hyoscine were given, but $n$-butylbro- mide hyoscine was suspended due to the appearance of blurred vision. Then i.v. fentanyl was attempted but with limited effect both in analgesia and duration of it. There were hours of the day in which the pain subsided by alone, and others in which it was very strong (with face scale $=10$ ) and unbearable. An enema gave negative result.

After an episode of aqueous vomiting, a direct abdomen radiography was performed which was negative for free air in the abdomen. Instead it showed presence of dolicocolon with faecal bulk in the right colon and sigma. The transverse colon was dilatated without air-water levels. The left colon was not displayed. Another enema was carried out, followed by evacuation of goat feces in the absence of mucus and blood.

The day after another abdominal ultrasound was performed due to the persistent symptomatology despite the therapy. The exam was unchanged compared to the previous one. First surgical consultation was requested. The surgeon didn't find any pathological signs neither peritonism, but he requested a barium enema.

*Corresponding author: Stefano Parmigiani, MD, PhD, Chief, Unit of Pediatrics and Neonatology, Eastern Liguria Hospital Sant'Andrea, Via V. Veneto, 197, 19124 - La Spezia, Italy

Accepted: November 19, 2019

Published online: November 21, 2019

Citation: Malcotti F, Scintu A, Nieri S, et al. (2019) A Recurrent Abdominal Pain with Chronic Constipation. Arch Pediatr Surg 3(1):59-61 


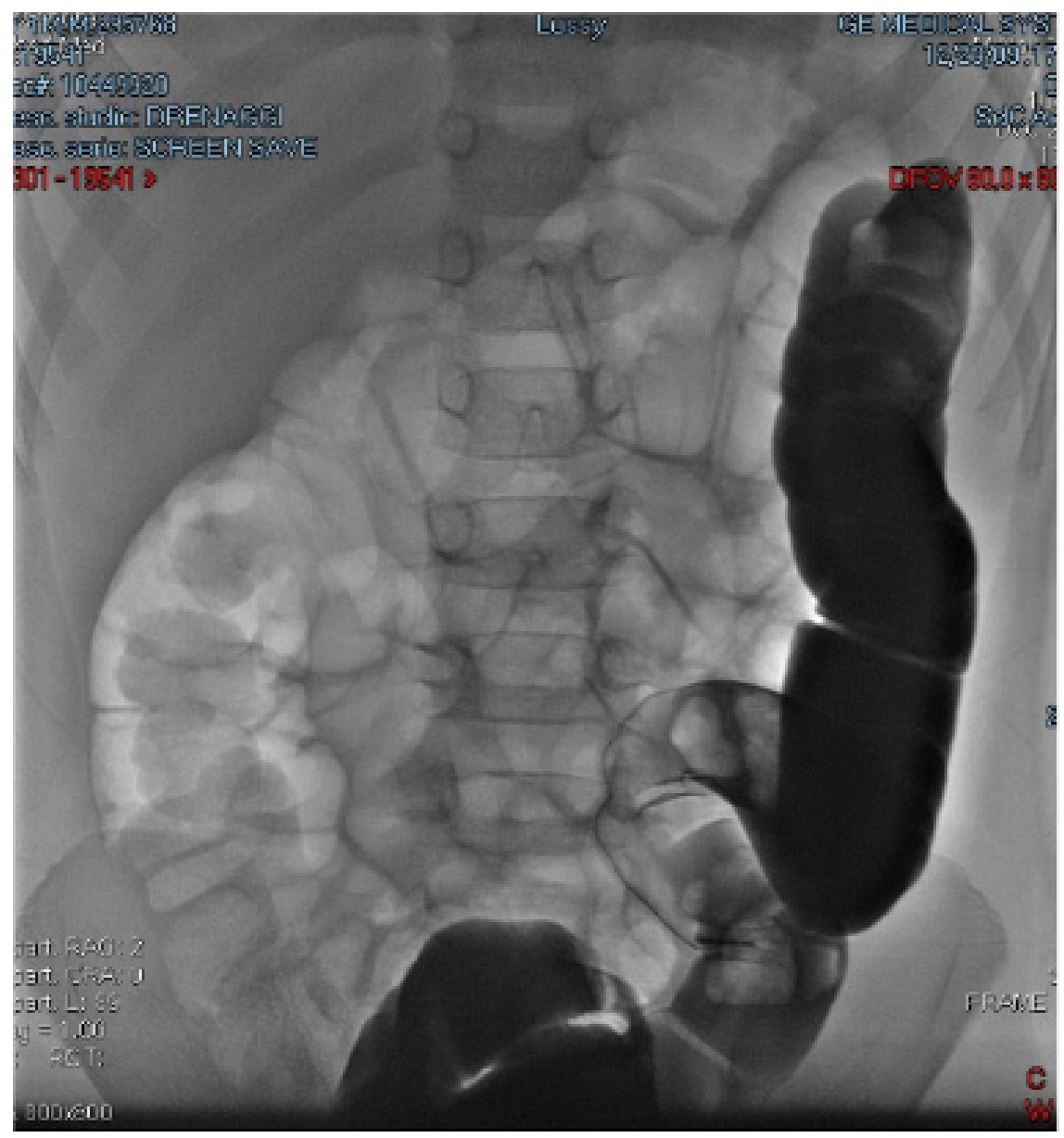

Figure 1: Note that contrast fills the left colon till to the left colonic flexure (black), while transverse and right colon are overdistended without air-liquid levels.

The examination was performed with Gastrografin ${ }^{\circledR}$. Even changing position to the baby various times to favour the passage of contrast medium, it did not pass the left colic flexure; the remaining colon was instead over distended by air after that point (Figure 1). The day after the patient had four episodes of bilious vomiting with meteoric and painful abdomen. Another ultrasound examination of the abdomen showed important gaseous distension in the upper quadrants, faecal stasis in the right colon with enlarged loops and the presence of modest liquid flap between the loops.

Further consultancy was required by the surgeon specialist who raised the suspicion of intestinal obstruction by volvulus or invagination, therefore diagnostic/therapeutic colonscopy and/or eventual laparoscopy were necessary. During the night before the foreseen examination, the child had a new episode of acute pain with bilious vomit. The child was therefore transferred to a pediatric hospital where pediatric surgeons were present. There he was promptly prepared for a laparoscopy that showed a diaphragmatic hernia of the left colon in the thoracic cavity.

After the intervention the child anyhow suffered abdominal pain and constipation. Other radiological exams showed normal diaphragm, but transverse colon remained dilated and this probably justify the persistence of constipation.

\section{Discussion}

Congenital diaphragmatic hernia $(C D H)$ is an early anomaly of fetal development that consists of an incomplete diaphragm formation with the simultaneous passage of the abdominal organs through the defect up into the thoracic cavity associated with abnormalities in lung development and function [1-3]. According to the anatomical position of the defect it is classified as: Bochdalek hernia (posterolateral defect), Morgagni hernia (anterior defect), total absence of the diaphragm, central hernia of the transverse septum, hiatal esophageal hernia [1-4]. The degree of defect severity is classified according to the characteristics and dimensions [5]. This is a rare condition: according to an European cohort study the prevalence is 1:3604 born live infants [6]. An even more rare condition is $\mathrm{CDH}$ with late presentation, which is diagnosed between the first month of life and 18-years, with a percentage between $2.6 \%$ and $20 \%$ of all cases of $\mathrm{CDH}$ [7]. Congenital diaphragmatic hernia can be associated in $40 \%$ of cases [1-3] (more frequently in the Morgagni type [8]) to other congenital cardiopulmonary malformations (tetralogy of Fallot, pulmonary hypoplasia), musculoskeletal and neurological malformations [9] or may occur in the context of specific syndromes (Beckwith-Wiedmann or chromosomal abnormalities such as trisomies 18 and 21 [2]. Early CDH 
is generally characterized by respiratory symptoms, such as tachypnea, dyspnea, cyanosis and respiratory failure at birth, while in the case of late presentation, gastrointestinal symptoms prevail. Symptoms like hyperacidity and reflux, recurrent vomiting, epigastric pain, constipation but also symptoms of intestinal obstruction are present especially in patients with a left diaphragmatic defect. In $10 \%$ of cases $\mathrm{CDH}$ can be completely asymptomatic [10]. Because of the different symptoms and the low frequency of pulmonary hypoplasia, late $\mathrm{CDH}$ results to have a better prognosis with reduced mortality $[11,12]$. Usually the diagnosis is prenatal through obstetric ultrasound, or occurs at birth in case of respiratory distress by chest X-ray showing abdominal contents in the thoracic cavity $[2,3,5]$. In some cases where the differential diagnosis with other thoracic malformations is more complex, gastrointestinal contrast agents are used [3]. Because of the rarity of the condition and the nonspecific symptoms, the definite diagnosis of $\mathrm{CDH}$ can be established after diagnostic-therapeutic approach only [13]. The therapy consists of surgery, to prevent complications such as stomach volvulus, perforation or strangulation of the herniated organ [14]. Previously, thoracoscopic or laparoscopic approaches were used, but now the minimally invasive surgical techniques are preferred. The latter appear to be burdened by a higher percentage of relapses which, however, are reduced if carried out by an expert team and if a prolonged monitoring of the patient is carried out [15-17].

\section{Conclusions}

This case report shows that in all cases of chronic gastrointestinal symptoms, such as vomiting, constipation and persistent abdominal pain, in which a consistent explanation cannot be found, the presence of a $\mathrm{CDH}$ should be taken into account in the differential diagnosis, even if the patient has an atypical age. Because of the rarity of the pathology and the patient's age, with periods of complete well-being alternating with periods of acute pain, in the present case the medical team carried out progressively more invasive diagnostic tools. The only element that could make one suspect an anomaly was the lack of visualization of the left colon either through the abdomen X-ray and with the barium enema.

A colon fibroscopy had already been arranged but the acuity of a crisis led the child to surgery without a definite diagnosis. It is truly important that such congenital malformation is kept in mind, even if very rare, when a clinical picture of recurrent abdominal pain cannot be otherwise explained. Probably a CT scan of the abdomen done without waiting, would give a more rapid answer, but the actual trend toward reduction of X-ray diagnostic and the rarity of the condition makes nowadays invasive investigations a non-primary diagnostic tool. Resolution of $\mathrm{CDH}$ after years in the present case had probably produced a persistent damage with low contractility of the over-distended bowel.

\section{Conflict of Interest}

The authors declare no conflict of interest.

\section{References}

1. McHoney M (2014) Congenital diaphragmatic hernia. Early Hum Dev 90: 941-946.

2. Leeuwen L, Fitzgerald DA (2014) Congenital diaphragmatic hernia. J Paediatr Child Health 50: 667-673.

3. Kotecha S, Barbato A, Bush A (2012) Congenital diaphragmatic hernia. Eur Respir J 39: 820-829.

4. Haroon J, Chamberlain RS (2013) An evidence-based review of the current treatment of congenital diaphragmatic hernia. Clin Pediatr 52: 115-124.

5. Lally KP, Lasky RE, Lally PA, et al. (2013) Standardized reporting for congenital diaphragmatic hernia: An international consensus. J Pediatr Surg 48: 2408-2415.

6. McGivern MR, Best KE, Rankin J, et al. (2015) Epidemiology of congenital diaphragmatic hernia in Europe: a register-based study. Arch Dis Child Fetal Neonatal Ed 100: 137-144.

7. Kitano Y, Lally KP, Lally PA, et al. (2005) Late-presenting congenital diaphragmatic hernia. J Pediatr Surg 40: 1839-1843.

8. Al-Salem AH, Zamakhshary Mal, Mohamed M, et al. (2014) Congenital morgagni's hernia: A national multicenter study. J Pediatr Surg 49: 503-507.

9. Bielinska M, Jay PY, Erlich JM, et al. (2007) Molecular genetics of congenital diaphragmatic defects. Ann Med 39: 261-274.

10. Bagłaj M, Dorobisz U (2005) Late-presenting congenital diaphragmatic hernia in children: A literature review. Pediatr Radiol 35: 478-488.

11. Chang S-W, Lee H-C, Yeung C-Y, et al. (2010) A twenty-year review of early and late-presenting congenital Bochdalek diaphragmatic hernia: Are they different clinical spectra? Pediatr Neonatol 51: 26-30.

12. Bagłaj M (2004) Late-presenting congenital diaphragmatic hernia in children: A clinical spectrum. Pediatr Surg Int 20: 658-669.

13. Alireza Malekzadegan, Alireza Sargazi (2016) Congenital diaphragmatic hernia with delayed presentation. Case Rep Surg.

14. Cigdem MK, Onen A, Otcu S, et al. (2007) Late presentation of bochdalek-type congenital diaphragmatic hernia in children: $A$ 23- year experience at a single center. Surg Today 37: 642-645.

15. Huang JS, Lau CT, Wong WY, et al. (2015) Thoracoscopic repair of congenital diaphragmatic hernia: Two centres' experience with 60 patients. Pediatr Surg Int 31: 191-195.

16. Tanaka T, Okazaki T, Fukatsu Y, et al. (2013) Surgical intervention for congenital diaphragmatic hernia: Open versus thoracoscopic surgery. Pediatr Surg Int 29: 1183-1186.

17. Liem NT, Nhat LQ, Tuan TM, et al. (2011) Thoracoscopic repair for congenital diaphragmatic hernia: Experience with 139 cases. J Laparoendosc Adv Surg Tech 21: 267-270.

DOI: $10.36959 / 472 / 354$

Copyright: (C) 2019 Malcotti F, et al. This is an open-access article distributed under the terms of the Creative Commons Attribution License, which permits unrestricted use, distribution, and reproduction in any medium, provided the original author and source are credited. 